\title{
Chaos Synchronization of a Finance Chaotic System with an Integral Sliding Mode Controller
}

\author{
Jingjing Wang and Chunzhi Yang (D) \\ Department of Mathematics, Huainan Normal University, Huainan 232038, China \\ Correspondence should be addressed to Chunzhi Yang; yangcz2002@126.com
}

Received 26 November 2020; Revised 29 December 2020; Accepted 6 January 2021; Published 20 January 2021

Academic Editor: Ahmed Mostafa Khalil

Copyright (C) 2021 Jingjing Wang and Chunzhi Yang. This is an open access article distributed under the Creative Commons Attribution License, which permits unrestricted use, distribution, and reproduction in any medium, provided the original work is properly cited.

In this paper, an integral sliding mode controller is proposed to realize the synchroniza-tion of a class of finance chaotic systems. First, an integral sliding surface with tanh function is designed. Then, fuzzy logic systems are used to estimate unknown functions, and then based on the Lyapunov stability theorem, the proposed control method can quickly drive the synchroniza-tion error into a small neighborhood of zero, and the range of this neighborhood can be estimated. The simulation results verify that the proposed method in this paper is better than the traditional method.

\section{Introduction}

In the past few decades, chaotic synchronization, due to its unique characteristics, has been widely used in engineering, finance, chemistry, confidential communications, and so on [1-5]. In recent decades, due to the complexity and inherent randomness of economic factors, chaotic nonlinear financial system has attracted extensive attention [6-12]. For example, Chao and Xingyuan [6] studied the existence of Hopf bifurcation and topological horseshoe of a class of finance chaotic system. Yu et al. [7] proposed a feedback control method to stabilize the hyperchaotic finance system to its equilibrium point. Huang and Cao [8] employed active control strategy to realize synchronization and antisynchronization of a class of fractional chaotic financial systems. In [11], a linear feedback control scheme was proposed to realize chaos synchronization of two identical financial chaotic systems. In addition to the feedback control method and active control method, there are other control methods that can realize synchronization of financial chaotic systems, such as adaptive control [13-15], impulsive control $[16,17]$, sliding mode control $[15,18-24]$, and so on.

Among above methods, more attention is paid to the sliding mode control method because of its simple design and good robustness. Sliding mode control is generally divided into two steps: the first step is to design the sliding mode surface; the second step is to design the controller so that the system state can enter the sliding mode surface. For example, Chiu [21] proposed two integral sliding mode control methods to make the tracking error reach the sliding surface in finite time. Using same two integral sliding modes, Zhao et al. [24] proposed two effective control methods, which inhibited the influence of external disturbances and realized the effective state estimation of the continuous stirred tank reactor. These two types of integral sliding mode are written as (1) $s=e+\lambda \int_{0}^{t} \operatorname{sign}(e) \mathrm{d} \tau$; (2) $s=e+\lambda \int_{0}^{t}|e|^{\gamma} \operatorname{sign}(e) \mathrm{d} \tau, \gamma \in(0,1), \lambda>0$. Since these integral sliding mode need to have sign $(\cdot)$ function, the controllers designed by them will lead to chattering phenomenon. Therefore, to modify the above integral sliding mode has become a research direction.

Inspired by the above works, this paper investigates the synchronization of two finance chaotic systems. The main contributions of this paper are summarized as follows.

(1) Compared with the traditional integral sliding mode, the integrated sliding mode proposed in this paper can estimate the ultimate synchronization error bound by its own dynamics.

(2) Compared with the traditional integral sliding mode control method, the proposed method can eliminate the chatter phenomenon in error states and controller. 
The rest of this work is arranged as follows. Some assumptions and lemmas and the problem statement are presented in Section 2. Section 3 gives sliding mode control design and stability analysis. In Section 4, some comparison results are presented to show the validity of the proposed method. At last, the conclusion is included in Section 5.

\section{Preliminaries}

In general, the finance chaotic system is described as

$$
\left\{\begin{array}{l}
\dot{x}_{1}=x_{3}+\left(x_{2}-\alpha_{1}\right) x_{1}, \\
\dot{x}_{2}=1-\beta_{1} x_{2}-x_{1}^{2}, \\
\dot{x}_{3}=-x_{1}-\sigma_{1} x_{3}
\end{array}\right.
$$

where $x=\left[x_{1}, x_{2}, x_{3}\right]^{T} \in R^{3}$ is the state vector, $x_{1}$ denotes the interest rate, $x_{2}$ denotes the investment demand, $x_{3}$ denotes the price exponent, parameter $\alpha_{1}$ denotes the saving amount, parameter $\beta_{1}$ denotes the investment cost, and parameter $\sigma_{1}$ denotes the elasticity of the demands of commercials. We regard system (1) as the master system, and the slave system is described as

$$
\left\{\begin{array}{l}
\dot{y}_{1}=y_{3}+\left(y_{2}-\alpha_{2}\right) y_{1}+d_{1}(t)+\operatorname{sat}\left(u_{1}\right) \\
\dot{y}_{2}=1-\beta_{2} y_{2}-y_{1}^{2}+d_{2}(t)+\operatorname{sat}\left(u_{2}\right) \\
\dot{y}_{3}=-y_{1}-\sigma_{2} y_{3}+d_{3}(t)+\operatorname{sat}\left(u_{3}\right)
\end{array}\right.
$$

where $y=\left[y_{1}, y_{2}, y_{3}\right]^{T} \in R^{3}$ is the state vector and $d(t)=$ $\left[d_{1}(t), d_{2}(t), d_{3}(t)\right]^{T}$ is the external disturbance vector. Parameters $\alpha_{2}, \beta_{2}$, and $\sigma_{2}$ are the same as $\alpha_{1}, \beta_{1}$, and $\sigma_{1}$ in system $(1) . \operatorname{sat}(u)=\left[\operatorname{sat}\left(u_{1}\right) \text {, sat }\left(u_{2}\right) \text {, sat }\left(u_{3}\right)\right]^{T}$ is control input, which is subject to saturation nonlinearity. And $\operatorname{sat}\left(u_{i}\right)$ is described as

$$
\operatorname{sat}\left(u_{i}\right)= \begin{cases}u_{i}^{b} \cdot \operatorname{sign}\left(u_{i}\right), & \text { if }\left|u_{i}\right| \geq u_{i}^{b}, \\ u_{i}, & \text { if }\left|u_{i}\right|<u_{i}^{b},\end{cases}
$$

where $u_{i}^{b}$ is the unknown bound of $u_{i}, i=1,2,3$. Define the synchronization error vector $e=y-x=\left[y_{1}-x_{1}, y_{2}-x_{2}\right.$, $\left.y_{3}-x_{3}\right]^{T}=\left[e_{1}, e_{2}, e_{3}\right]^{T}$. According to (1) and (2), we obtain the following error dynamic system:

$$
\left\{\begin{array}{l}
\dot{e}_{1}=g_{1}(y)-f_{1}(x)+d_{1}(t)+\operatorname{sat}\left(u_{1}\right), \\
\dot{e}_{2}=g_{2}(y)-f_{2}(x)+d_{2}(t)+\operatorname{sat}\left(u_{2}\right), \\
\dot{e}_{3}=g_{3}(y)-f_{3}(x)+d_{3}(t)+\operatorname{sat}\left(u_{3}\right),
\end{array}\right.
$$

where $f_{1}(x)=x_{3}+\left(x_{2}-\alpha_{1}\right) x_{1}, g_{1}(y)=y_{3}+\left(y_{2}-\alpha_{2}\right) y_{1}$, $f_{2}(x)=1-\beta_{1} x_{2}-x_{1}^{2}, g_{2}(y)=1-\beta_{2} y_{2}-x_{2}^{2}$,

$f_{3}(x)=-x_{1}-\sigma_{1} x_{3}, g_{3}(y)=-y_{1}-\sigma_{2} y_{3}$.

In order to achieve effective state synchronization between the master chaotic system and the slave chaotic system, we make the following assumptions about $f_{i}(x)$, $g_{i}(y)$, and $d_{i}(t), i=1,2,3$.

Assumption 1. Nonlinear functions $f_{i}(x)$ and $g_{i}(y)$ are unknown but bounded, and states $x$ and $y$ are measurable.

Assumption 2. The external disturbance $d_{i}(t)$ is bounded, i.e., $\left|d_{i}(t)\right| \leq d_{i}^{*}$, where $d_{i}^{*}$ is unknown.
Remark 1. In [11], the linear feedback control method is used to synchronize two identical finance chaotic systems, but the unknown function and saturation nonlinearity input of the finance chaotic system are not considered. At the same time, in order to make the controller have good robustness, an integral sliding mode control method is used to discuss the synchronization of master system (1) and slave system (2).

Remark 2. Fuzzy logic systems are used in this paper to estimate unknown functions $f_{i}(x)$ and $g_{i}(y)$, and the related fuzzy estimation theory can be found in the literature [25]. In addition, $d_{i}^{*}$ is only used in stability analysis and not involved in the design of $u_{i}$.

By using FLSs, $f_{i}(x)$ and $g_{i}(y)$ can be approximated as

$$
\begin{array}{ll}
f_{i}(x)=\theta_{f_{i}}^{* T} \varphi_{f_{i}}(x)+\varepsilon_{f_{i}}, & \left|\varepsilon_{f_{i}}\right| \leq \varepsilon_{f_{i}}^{*}, \\
g_{i}(y)=\theta_{g_{i}}^{* T} \varphi_{g_{i}}(y)+\varepsilon_{g_{i}}, & \left|\varepsilon_{g_{i}}\right| \leq \varepsilon_{g_{i}}^{*},
\end{array}
$$

where $\theta_{f_{i}}^{*}, \theta_{g_{i}}^{*} \in R^{m_{i}}$ are ideal weight vectors, $\varepsilon_{f_{i}}$ and $\varepsilon_{g_{i}}$ are approximation errors, and $\varepsilon_{f_{i}}^{*}$ and $\varepsilon_{g_{i}}^{*}$ are upper bounds of $\varepsilon_{f_{i}}$ and $\varepsilon_{g_{i}}$, respectively. $\varphi_{f_{i}}(x)$ and $\varphi_{g_{i}}(y)$ are basis functions.

Define $\widetilde{\theta}_{f_{i}}=\theta_{f_{i}}^{*}-\widehat{\theta}_{f_{i}}, \widetilde{\theta}_{g_{i}}=\theta_{g_{i}}^{*}-\widehat{\theta}_{g_{i}}$ where $\widehat{\theta}_{f_{i}}$ and $\widehat{\theta}_{g_{i}}$ are the estimations of $\theta_{f_{i}}^{*}$ and $\theta_{g_{i}}^{*}$, respectively. So, we obtain

$$
\begin{aligned}
& f_{i}(x)=\tilde{\theta}_{f_{i}}^{T} \varphi_{f_{i}}(x)+\widehat{\theta}_{f_{i}}^{T} \varphi_{f_{i}}(x)+\varepsilon_{f_{i}}(x), \\
& g_{i}(y)=\tilde{\theta}_{g_{i}}^{T} \varphi_{g_{i}}(y)+\widehat{\theta}_{g_{i}}^{T} \varphi_{g_{i}}(y)+\varepsilon_{g_{i}}(y) .
\end{aligned}
$$

Substituting (7) into (4), error system (4) can be rewritten as

$$
\left\{\begin{array}{l}
\dot{e}_{1}=\tilde{\theta}_{g_{1}}^{T} \varphi_{g_{1}}(y)+\hat{\theta}_{g_{1}}^{T} \varphi_{g_{1}}(y)-\tilde{\theta}_{f_{1}}^{T} \varphi_{f_{1}}(x)-\hat{\theta}_{f_{1}}^{T} \varphi_{f_{1}}(x)+\bar{d}_{1}+u_{1}, \\
\dot{e}_{2}=\tilde{\theta}_{g_{2}}^{T} \varphi_{g_{2}}(y)+\hat{\theta}_{g_{2}}^{T} \varphi_{g_{2}}(y)-\tilde{\theta}_{f_{2}}^{T} \varphi_{f_{2}}(x)-\hat{\theta}_{f_{2}}^{T} \varphi_{f_{2}}(x)+\bar{d}_{2}+u_{2}, \\
\dot{e}_{3}=\tilde{\theta}_{g_{3}}^{T} \varphi_{g_{3}}(y)+\widehat{\theta}_{g_{3}}^{T} \varphi_{g_{3}}(y)-\tilde{\theta}_{f_{3}}^{T} \varphi_{f_{3}}(x)-\widehat{\theta}_{f_{3}}^{T} \varphi_{f_{3}}(x)+\bar{d}_{3}+u_{3},
\end{array}\right.
$$

where $\bar{d}_{i}=\varepsilon_{g_{i}}(y)-\varepsilon_{f_{i}}\left(\underline{x}_{*}\right)+d_{i}(t)+\Delta \underline{u}_{i}, \Delta \underline{u}_{i}=\operatorname{sat}\left(u_{i}\right)-u_{i}$. Obviously, there exists $\bar{d}_{i}^{*}$ such that $\left|\bar{d}_{i}\right| \leq \bar{d}_{i}^{*}$.

The following lemma is introduced for the subsequent discussions.

Lemma 1 (see [14]). For any $z \in R, 1>\gamma \geq 0$, and $\varepsilon>0$, the following inequality holds:

$$
|z|^{\gamma+1}-z|z|^{\gamma} \tanh \left(\frac{z}{\varepsilon}\right) \leq \eta_{\gamma} \varepsilon^{\gamma+1},
$$

where $\eta_{\gamma}=\varrho^{\gamma}(2 \varrho-\gamma-1)>0$ and $\varrho$ is the unique solution of $\varrho(1+\tanh (\varrho))=\gamma+1$.

Remark 3. In this paper, inequality (9) of Lemma 1 is used to discuss the ultimate boundary of tracking error $e_{i}$.

The aim of this paper is to design a novel integral sliding variable such that the error state $e$ reaches a small neighborhood of zero quickly. 


\section{Sliding Mode Control Design and Stability Analysis}

In order to design a sliding mode robust controller to make the synchronization error $e$ stable, an integral sliding variable needs to be designed. In this paper, we design the sliding mode as follows:

$$
\left\{\begin{array}{l}
s_{1}=e_{1}+\int_{0}^{t}\left(\mu_{1} \operatorname{Tanh}_{\varepsilon_{1}}^{\gamma_{1}}\left(e_{1}\right)+\mu_{2} \operatorname{Tanh}_{\varepsilon_{1}}^{0}\left(e_{1}\right)\right) \mathrm{d} \tau \\
s_{2}=e_{2}+\int_{0}^{t}\left(\mu_{1} \operatorname{Tanh}_{\varepsilon_{1}}^{\gamma_{1}}\left(e_{2}\right)+\mu_{2} \operatorname{Tanh}_{\varepsilon_{1}}^{0}\left(e_{2}\right)\right) \mathrm{d} \tau \\
s_{3}=e_{3}+\int_{0}^{t}\left(\mu_{1} \operatorname{Tanh}_{\varepsilon_{1}}^{\gamma_{1}}\left(e_{3}\right)+\mu_{2} \operatorname{Tanh}_{\varepsilon_{1}}^{0}\left(e_{3}\right)\right) \mathrm{d} \tau
\end{array}\right.
$$

where $\operatorname{Tanh}_{\varepsilon_{1}}^{\gamma_{1}}\left(e_{i}\right)=\left|e_{i}\right|^{\gamma_{i}} \tanh \left(e_{i} / \varepsilon_{1}\right), \quad \operatorname{Tanh}_{\varepsilon_{1}}^{0}\left(e_{i}\right)=\tanh$ $\left(e_{i} / \varepsilon_{1}\right), \mu_{1}, \mu_{2}$ are designed positive parameters, $\gamma_{1} \in(0,1)$, and $\varepsilon_{1}$ is a small positive constant, $i=1,2,3$.

Remark 4. Different from traditional sliding mode control method that the occurrence of chatter phenomenon is mainly due to the hysteresis of control switching, the integral sliding variable (10) used in this paper can avoid chatter phenomenon and estimate the ultimate boundary of the tracking error $e$.

According to (8) and (10), the time derivative of $s_{i}$ is obtained as follows:

$$
\begin{aligned}
\dot{s}_{i}= & \widetilde{\theta}_{g_{i}}^{T} \varphi_{g_{i}}(y)+\widehat{\theta}_{g_{i}}^{T} \varphi_{g_{i}}(y)-\widetilde{\theta}_{f_{i}}^{T} \varphi_{f_{i}}(x)-\widehat{\theta}_{f_{i}}^{T} \varphi_{f_{i}}(x)+\bar{d}_{i}(t) \\
& +u_{i}+\mu_{1} \operatorname{Tanh}_{\epsilon_{1}}^{\gamma_{1}}\left(e_{i}\right)+\mu_{2} \operatorname{Tanh}_{\epsilon_{1}}^{0}\left(e_{i}\right) .
\end{aligned}
$$

Based on the above analysis, we give the main result as follows.
Theorem 1. Consider the synchronization error system (8) with the external disturbance; if the integral sliding variable is constructed as (10) and the controller $u_{i}$ is designed as

$$
\begin{aligned}
u_{i}= & -\mu_{3} s_{i}-\mu_{4} \operatorname{Tanh}_{\epsilon_{2}}^{\gamma_{2}}\left(s_{i}\right)-\widehat{\theta}_{g_{i}}^{T} \varphi_{g_{i}}(y)+\widehat{\theta}_{f_{i}}^{T} \varphi_{f_{i}}(x) \\
& -\mu_{1} \operatorname{Tanh}_{\epsilon_{1}}^{\gamma_{1}}\left(e_{i}\right)-\mu_{2} \operatorname{Tanh}_{\epsilon_{1}}^{0}\left(e_{i}\right),
\end{aligned}
$$

and parameter adaptive laws are proposed as

$$
\begin{aligned}
& \dot{\hat{\theta}}_{g_{i}}=\eta_{g}\left[s_{i} \varphi_{g_{i}}(y)-\delta_{1} \widehat{\theta}_{g_{i}}\right], \\
& \dot{\hat{\theta}}_{f_{i}}=\eta_{f}\left[-s_{i} \varphi_{f_{i}}(x)-\delta_{2} \widehat{\theta}_{f_{i}}\right],
\end{aligned}
$$

where $\operatorname{Tanh}_{\epsilon_{2}}^{\gamma_{2}}\left(s_{i}\right)=\left|s_{i}\right|^{\gamma_{2}} \tanh \left(s_{i} / \varepsilon_{2}\right), \delta_{1}, \delta_{2}, \mu_{3}, \mu_{4}$, and $\eta_{g}, \eta_{f}$ are design positive parameters, $i=1,2,3$, and $\varepsilon_{2}$ is a small positive constant, then all the closed-loop system signals in (14) are uniformly ultimately bounded.

Proof. Consider the Lyapunov function as

$$
V=V_{1}+V_{2}+V_{3}
$$

where

$$
\begin{aligned}
& V_{1}=\sum_{i=1}^{3} \frac{1}{2} s_{i}^{2}, \\
& V_{2}=\sum_{i=1}^{3} \frac{1}{2 \eta_{g}} \tilde{\theta}_{g_{i}}^{T} \tilde{\theta}_{g_{i}}, \\
& V_{3}=\sum_{i=1}^{3} \frac{1}{2 \eta_{f}} \tilde{\theta}_{f_{i}}^{T} \tilde{\theta}_{f_{i}} .
\end{aligned}
$$

Substituting (12) into (11), the derivative of $V_{1}$ can be obtained as

$$
\begin{aligned}
& \dot{V}_{1}=\sum_{i=1}^{3} s_{i} \dot{s}_{i} \\
&=\sum_{i=1}^{3} s_{i}\left(\tilde{\theta}_{g_{i}}^{T} \varphi_{g_{i}}(y)+\widehat{\theta}_{g_{i}}^{T} \varphi_{g_{i}}(y)-\tilde{\theta}_{f_{i}}^{T} \varphi_{f_{i}}(x)-\widehat{\theta}_{f_{i}}^{T} \varphi_{f_{i}}(x)+\bar{d}_{i}(t)+u_{i}+\mu_{1} \operatorname{Tanh}_{\epsilon_{1}}^{\gamma_{1}}\left(e_{i}\right)+\mu_{2} \operatorname{Tanh}_{\epsilon_{1}}^{0}\left(e_{i}\right)\right) \\
&=\sum_{i=1}^{3} s_{i}\left(\tilde{\theta}_{g_{i}}^{T} \varphi_{g_{i}}(y)-\widehat{\theta}_{f_{i}}^{T} \varphi_{f_{i}}(x)-\mu_{3} s_{i}-\mu_{4} \operatorname{Tanh}_{\epsilon_{2}}^{\gamma_{2}}\left(s_{i}\right)+\bar{d}_{i}(t)\right) \\
&=-\mu_{3} \sum_{i=1}^{3} s_{i}^{2}-\sum_{i=1}^{n} \mu_{4} s_{i}\left|s_{i}\right|^{\gamma_{2}} \tanh \left(\frac{s_{i}}{\epsilon_{2}}\right)+\sum_{i=1}^{3} s_{i} \bar{d}_{i}(t)+\sum_{i=1}^{3} s_{i} \tilde{\theta}_{g_{i}}^{T} \varphi_{g}(y)-\sum_{i=1}^{3} s_{i} \tilde{\theta}_{f_{i}}^{T} \varphi_{f}(x) \\
& \dot{V}_{2}=\sum_{i=1}^{3} \frac{1}{\eta_{g 3}} \tilde{\theta}_{g_{i}}^{T} \dot{\tilde{\theta}}_{g_{i}}=\sum_{i=1}^{3}\left[-s_{i} \tilde{\theta}_{g_{i}}^{T} \varphi_{g_{i}}(y)+\delta_{1} \tilde{\theta}_{g_{i}}^{T} \widehat{\theta}_{g_{i}}\right]
\end{aligned}
$$

From (13), the time derivative of $V_{2}$ and $V_{3}$ yields

$$
\dot{V}_{3}=\sum_{i=1}^{3} \frac{1}{\eta_{f}} \widetilde{\theta}_{f_{i}}^{T} \dot{\tilde{\theta}}_{f_{i}}=\sum_{i=1}^{3}\left[s_{i} \widetilde{\theta}_{f_{i}}^{T} \varphi_{f_{i}}(x)+\delta_{2} \widetilde{\theta}_{f_{i}}^{T} \widehat{\theta}_{f_{i}}\right] .
$$

Using (16), (17), and (18), one gets 


$$
\begin{aligned}
\dot{V}= & -\mu_{3} \sum_{i=1}^{3} s_{i}^{2}-\sum_{i=1}^{3} \mu_{4} s_{i}\left|s_{i}\right| \gamma^{\gamma_{2}} \tanh \left(\frac{s_{i}}{\varepsilon_{2}}\right)+\sum_{i=1}^{3} s_{i} \bar{d}_{i}(t) \\
& +\sum_{i=1}^{3} \delta_{1} \widetilde{\theta}_{g_{i}}^{T} \widehat{\theta}_{g_{i}}+\sum_{i=1}^{3} \delta_{2} \widetilde{\theta}_{f_{i}}^{T} \widehat{\theta}_{f_{i}} .
\end{aligned}
$$

Since the following inequalities hold:

$$
\begin{gathered}
s_{i} \bar{d}_{i}(t) \leq \frac{s_{i}^{2}}{2}+\frac{\bar{d}_{i}^{2}(t)}{2} \leq \frac{s_{i}^{2}}{2}+\frac{\bar{d}_{i}^{* 2}(t)}{2}, \\
\delta_{1} \widetilde{\theta}_{g_{i}}^{T} \widehat{\theta}_{g_{i}} \leq-\frac{\delta_{1}}{2}\left\|\widetilde{\theta}_{g_{i}}\right\|^{2}+\frac{\delta_{1}}{2}\left\|\theta_{g_{i}}^{*}\right\|^{2}, \\
\delta_{2} \widetilde{\theta}_{f_{i}}^{T} \widehat{\theta}_{f_{i}} \leq-\frac{\delta_{2}}{2}\left\|\widetilde{\theta}_{f_{i}}\right\|^{2}+\frac{\delta_{2}}{2}\left\|\theta_{f_{i}}^{*}\right\|^{2},
\end{gathered}
$$

by using Lemma 1 , one has

$$
-\sum_{i=1}^{3} \mu_{4} s_{i}\left|s_{i}\right|^{\gamma_{2}} \tanh \left(\frac{s_{i}}{\varepsilon_{2}}\right) \leq-\sum_{i=1}^{3} \mu_{4}\left|s_{i}\right|^{\gamma_{2}+1}+3 \mu_{4} \eta_{\gamma_{2}} \varepsilon_{2}^{\gamma_{2}+1} .
$$

Substituting (20) and (21) into (19) results in

$$
\begin{aligned}
\dot{V} \leq & -\sum_{i=1}^{3}\left(\mu_{3}-\frac{1}{2}\right) s_{i}^{2}-\sum_{i=1}^{3} \mu_{4}\left|s_{i}\right|^{\gamma_{2}+1} \\
& -\frac{\delta_{1}}{2} \sum_{i=1}^{3}\left\|\tilde{\theta}_{g_{i}}\right\|^{2}-\frac{\delta_{2}}{2} \sum_{i=1}^{3}\left\|\widetilde{\theta}_{f_{i}}\right\|^{2}+r_{0},
\end{aligned}
$$

where $r_{0}=\sum_{i=1}^{3}\left(\bar{d}_{i}^{* 2} / 2+3 \mu_{4} \eta_{\gamma_{2}} \varepsilon_{2}^{\gamma_{2}+1}\right)+\left(\delta_{2} / 2\right) \sum_{i=1}^{3}\left\|\theta_{g_{i}}^{*}\right\|^{2}+$ $\left(\delta_{2} / 2\right) \sum_{i=1}^{3}\left\|\theta_{f_{i}}^{*}\right\|^{2}$. And let $r_{\text {min }}=\min \left\{2 \mu_{3}-1, \eta_{g} \delta_{1}, \eta_{f} \delta_{2}\right\}$. It can be obtained as

$$
\dot{V} \leq-r_{\min } V+r_{0} .
$$

From (23), we know that

$$
V \leq \frac{r_{0}}{r_{\min }}+\left(V(0)-\frac{r_{0}}{r_{\min }}\right) e^{-r_{\min } t} \leq 2\left(V(0)+\frac{r_{0}}{r_{\min }}\right) .
$$

Obviously, it can be concluded that all signals in (14) are ultimately uniformly bounded. The proof is completed.

Theorem 1 shows that the designed sliding mode controller (12) guarantees that all signals in (14) are bounded. Now, we further study the convergence of the synchronization error $e_{i}$. According to (10), (11), and (12), we have

$$
\begin{aligned}
\dot{e}_{i} & =\dot{s}_{i}-\mu_{1} \operatorname{Tanh}_{\epsilon_{1}}^{\gamma_{1}}\left(e_{i}\right)-\mu_{2} \operatorname{Tanh}_{\epsilon_{1}}^{0}\left(e_{i}\right) \\
& =\psi_{i}(t)-\mu_{1} \operatorname{Tanh}_{e_{1}}^{\gamma_{1}}\left(e_{i}\right)-\mu_{2} \operatorname{Tanh}_{\epsilon_{1}}^{0}\left(e_{i}\right),
\end{aligned}
$$

where $\psi_{i}(t)=\tilde{\theta}_{g_{i}}^{T} \varphi_{g_{i}}(y)-\tilde{\theta}_{f_{i}}^{T} \varphi_{f_{i}}(x)+\bar{d}_{i}(t)-\mu_{3} s_{i}-\mu_{4}$ Tanh $\gamma_{2}\left(s_{i}\right)$. Through the conclusion of Theorem 1 , there exists a positive constant $\varpi_{i}$ such that

$$
\left|\psi_{i}(t)\right| \leq \emptyset_{i} .
$$

Consider the following Lyapunov function:

$$
\bar{V}=\frac{1}{2} \sum_{i=1}^{3} e_{i}^{2} .
$$

The derivative of $\bar{V}$ is calculated as

$$
\begin{aligned}
\dot{\bar{V}}= & \sum_{i=1}^{3}\left(e_{i} \psi_{i}(t)-\mu_{1} e_{i} \operatorname{Tanh}_{\epsilon_{1}}^{\gamma_{1}}\left(e_{i}\right)-\mu_{2} e_{i} \operatorname{Tanh}_{\epsilon_{1}}^{0}\left(e_{i}\right)\right) \\
\leq & \sqrt{e_{1}^{2}+e_{2}^{2}+e_{3}^{2}} \sqrt{\omega_{1}^{2}+\omega_{2}^{2}+\omega_{3}^{2}}-\sum_{i=1}^{3} \mu_{1} e_{i}\left|e_{i}\right|^{\gamma_{1}} \tanh \left(\frac{e_{i}}{\epsilon_{1}}\right) \\
& -\sum_{i=1}^{3} \mu_{2} e_{i} \tanh \left(\frac{e_{i}}{\epsilon_{1}}\right) .
\end{aligned}
$$

By using Lemma 1, the following inequalities hold:

$$
\begin{aligned}
-\sum_{i=1}^{3} \mu_{1} e_{i}\left|e_{i}\right|^{\gamma_{1}} \tanh \left(\frac{e_{i}}{\varepsilon_{1}}\right) \leq & -\sum_{i=1}^{3} \mu_{1}\left|e_{i}\right|^{\gamma_{1}+1}+3 \mu_{1} \eta_{\gamma_{1}} \varepsilon_{1}^{\gamma_{1}+1} \\
\leq & -\mu_{1}\left(e_{1}^{2}+e_{2}^{2}+e_{3}^{2}\right)^{\left(\gamma_{1}+1\right) / 2} \\
& +3 \mu_{1} \eta_{\gamma_{1}} \varepsilon_{1}^{\gamma_{1}+1}, \\
-\sum_{i=1}^{n} \mu_{2} e_{i} \tanh \left(\frac{e_{i}}{\varepsilon_{1}}\right) \leq & -\sum_{i=1}^{n} \mu_{2}\left|e_{i}\right|+3 \mu_{2} \eta_{\gamma_{0}} \varepsilon_{1} \\
\leq & -\mu_{2} \sqrt{e_{1}^{2}+e_{2}^{2}+e_{3}^{2}}+3 \mu_{2} \eta_{\gamma_{0}} \varepsilon_{1} .
\end{aligned}
$$

Substituting (29) and (30) into (28), we have

$\dot{\bar{V}} \leq-\left(\mu_{2}-\bar{\Phi}\right) \sqrt{e_{1}^{2}+e_{2}^{2}+e_{3}^{2}}-\mu_{1}\left(e_{1}^{2}+e_{2}^{2}+e_{3}^{2}\right)^{\left(\gamma_{1}+1\right) / 2}+\varepsilon_{0}$,

where $\quad \bar{\omega}=\sqrt{\omega_{1}^{2}+\omega_{2}^{2}+\omega_{3}^{2}}, \varepsilon_{0}=3 \mu_{1} \eta_{\gamma_{1}} \varepsilon_{1}^{\gamma_{1}+1}+3 \mu_{2} \eta_{\gamma_{0}} \varepsilon_{1}$. Selecting $\mu_{2}$ satisfies $\mu_{2}>\bar{\Phi}$, and define the compact set as

$$
\Omega_{e}=\left\{e \in R^{3} \mid \mu_{1}\left(e_{1}^{2}+e_{2}^{2}+e_{3}^{2}\right)^{\gamma_{1}+1 / 2} \leq \frac{\varepsilon_{0}}{1-\varsigma}\right\},
$$

where $\varsigma \in(0,1)$ is any number. If $e \notin \Omega_{e}$, then

$$
\begin{aligned}
\dot{\bar{V}} & \leq-\mu_{1}\left(e_{1}^{2}+e_{2}^{2}+e_{3}^{2}\right)^{\gamma+1 / 2}+\varepsilon_{0}<-\mu_{1} \varsigma\left(e_{1}^{2}+e_{2}^{2}+e_{3}^{2}\right)^{\gamma_{1}+1 / 2} \\
& =-2^{\gamma_{1}+1 / 2} \mu_{1} \varsigma \bar{V}^{\gamma_{1}+1 / 2} .
\end{aligned}
$$

Obviously, $\bar{V}$ decreases monotonically outside the set $\Omega_{\mathrm{e}}$ until it enters the minimal level set of $\bar{V}$ containing $\Omega_{e}$. Therefore, we obtain the following theorem.

Theorem 2. Based on the conditions and results of Theorem 1 and selecting the appropriate parameters $\mu_{1}$ and $\mu_{2}$ in (10), the 


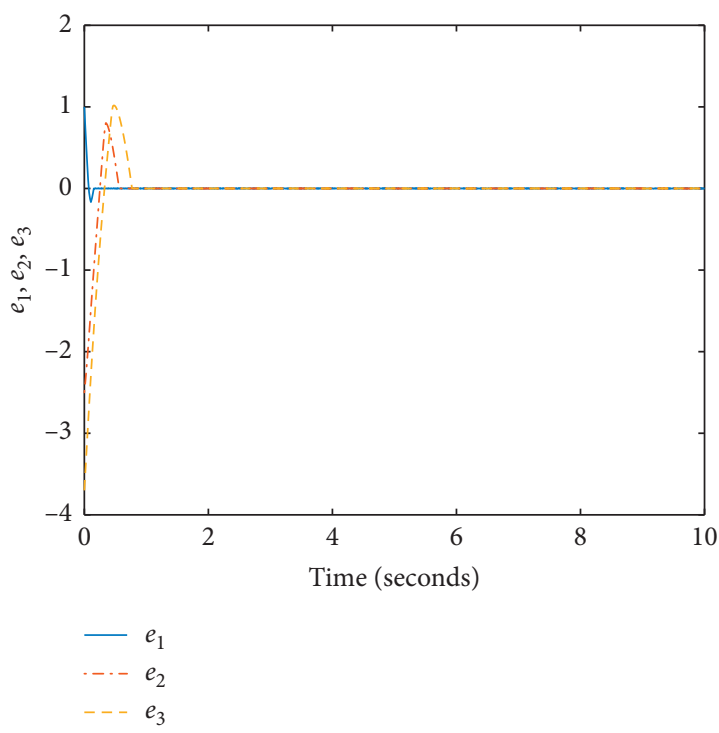

(a)

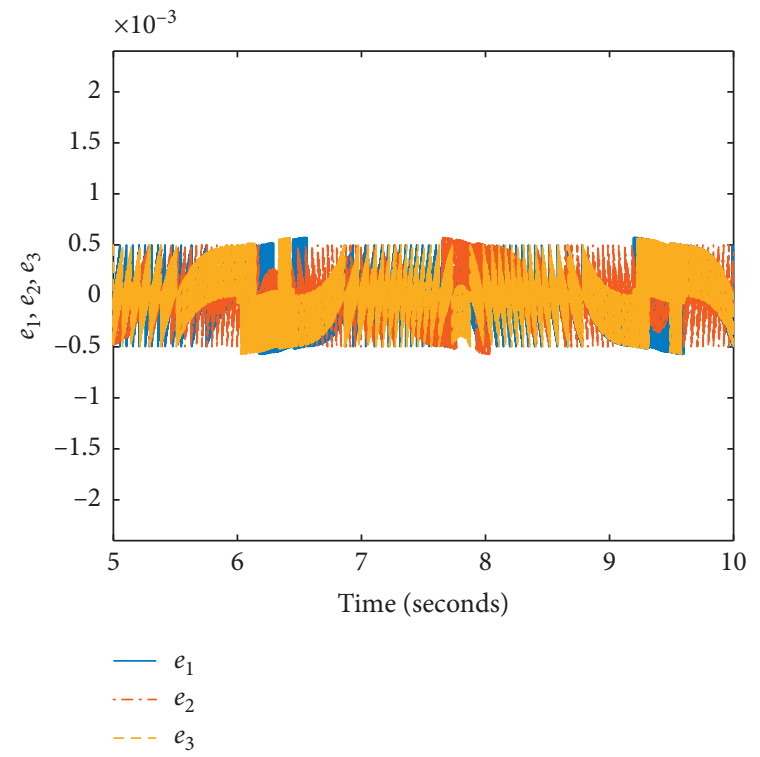

(b)

Figure 1: The trajectories of tracking errors $e_{1}, e_{2}, e_{3}$ by method (35) in time period (a) $[0,10 \mathrm{~s}]$ and (b) [5s, $\left.10 \mathrm{~s}\right]$.

tracking error vector $e$ is ultimately bounded and the ultimate bound is $\|e\| \leq\left(\varepsilon_{0} / \mu_{1}(1-\varsigma)\right)^{1 / \gamma_{1}+1}$.

Remark 5. The synchronization method of chaotic systems in this paper can be extended to generalized projective synchronization, lag synchronization, and so on.

\section{Synchronization of Finance Chaotic System}

In this section, the chaotic finance system [11] is introduced to demonstrate the effectiveness of the proposed control method (12). Parameters in the master system (1) and the slave system (2) are selected as $\alpha_{1}=0.8, \alpha_{2}=0.9$, $\beta_{1}=0.2, \beta_{2}=0.25, \sigma_{1}=1.9, \sigma_{2}=1.8$, and external disturbances $d_{1}(t)=2.0 \sin (t), d_{2}(t)=2.0 \cos (2 t), d_{3}(t)=2.0$ $\sin (3 t)$. Here, initial values of $x$ and $y$ are chosen as $x(0)=$ $[1,1.5,1.2]^{T}$ and $y(0)=[2,-1,-2.5]^{T}$, and other parameters are chosen as $\eta_{g}=\eta_{f}=50, \mu_{1}=\mu_{2}=\mu_{3}=\mu_{4}=5$, $\varsigma=1 / 7, \varepsilon_{1}=\varepsilon_{2}=0.005, \gamma_{1}=1 / 5, \gamma_{2}=1 / 5, u_{1}^{b}=u_{2}^{b}=u_{3}^{b}=8$, $\delta_{1}=\delta_{2}=0.01$. The fuzzy membership functions are selected as

$$
\begin{aligned}
& \theta_{f_{1}}(\zeta)=\exp \left[-\frac{1}{2}\left(\frac{\zeta+4.5-1.5 k_{1}}{0.7}\right)^{2}\right] \\
& \theta_{g_{1}}(x i)=\exp \left[-\frac{1}{2}\left(\frac{\xi+4.5-1.5 k_{1}}{0.7}\right)^{2}\right], \\
& \theta_{f_{2}}(\zeta)=\exp \left[-\frac{1}{2}\left(\frac{\zeta+5.5-1.5 k_{1}}{0.7}\right)^{2}\right], \\
& \theta_{g_{2}}(\xi)=\exp \left[-\frac{1}{2}\left(\frac{\xi+5.5-1.5 k_{1}}{0.7}\right)^{2}\right], \\
& \theta_{f_{3}}(\zeta)=\exp \left[-\frac{1}{2}\left(\frac{\zeta+3.0-1.5 k_{2}}{0.7}\right)^{2}\right], \\
& \theta_{g_{3}}(\xi)=\exp \left[-\frac{1}{2}\left(\frac{\xi+3.0-1.5 k_{2}}{0.7}\right)^{2}\right],
\end{aligned}
$$




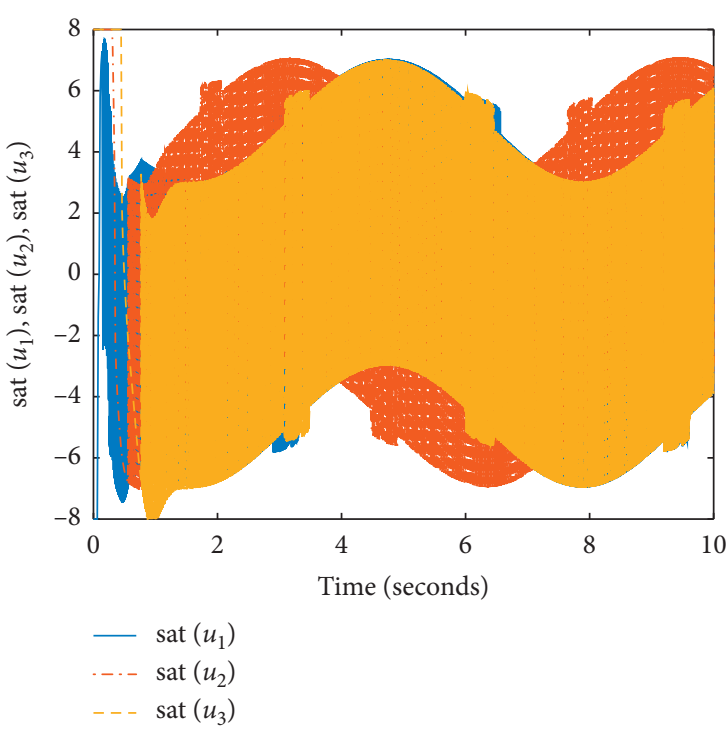

(a)

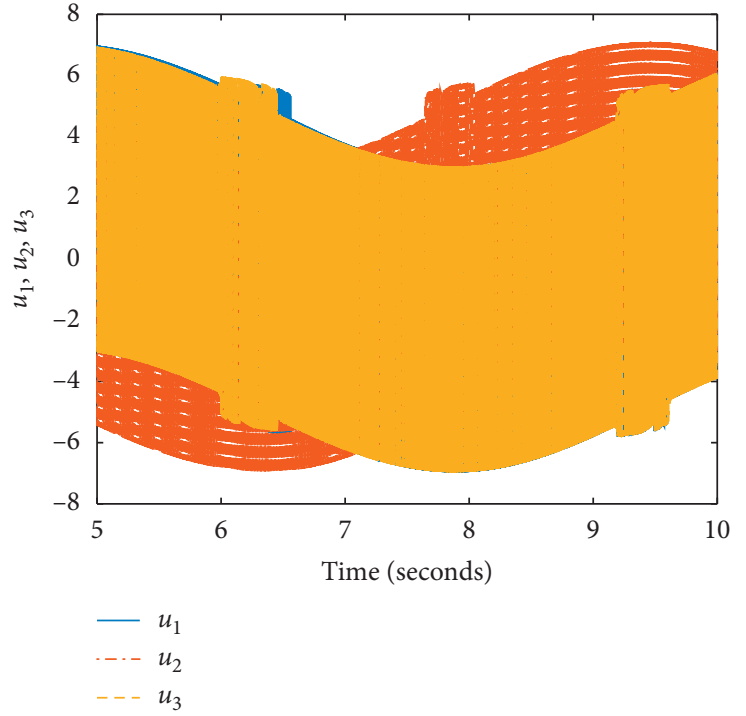

(b)

Figure 2: The trajectories of (a) sat $\left(u_{1}\right)$, sat $\left(u_{2}\right)$, sat $\left(u_{3}\right)$ by method (35) in time period $[0,10 \mathrm{~s}]$ and (b) $u_{1}, u_{2}, u_{3}$ by method $(35)$ in time period $[5 \mathrm{~s}, 10 \mathrm{~s}]$.

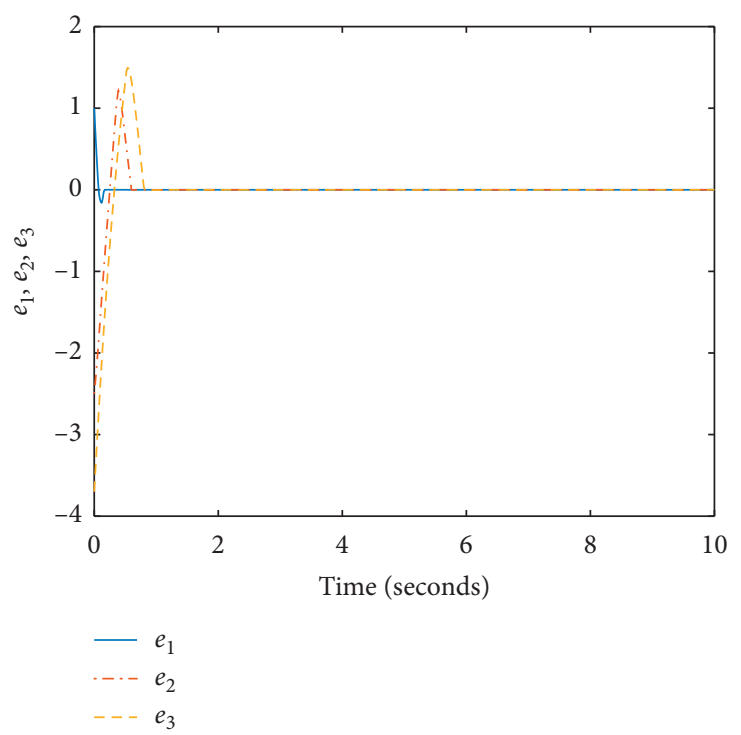

(a)

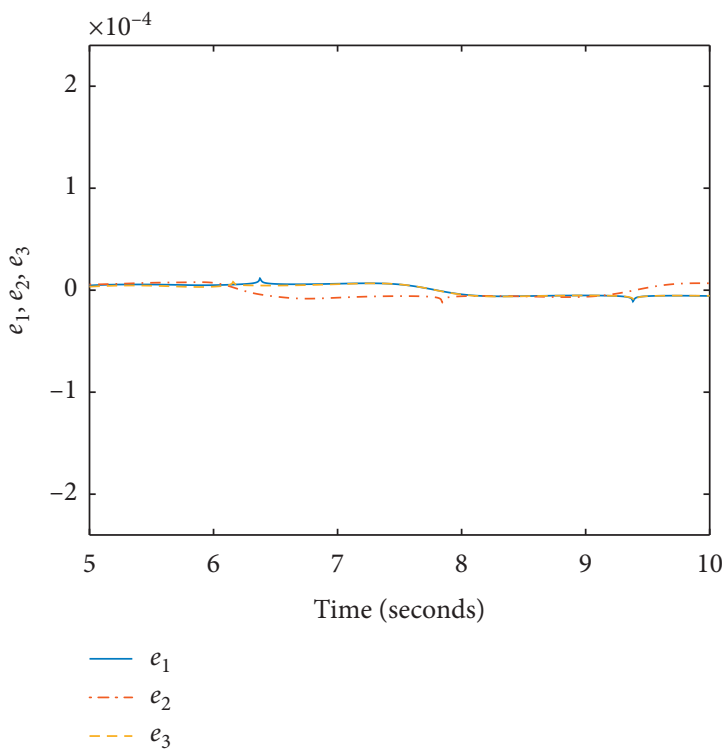

(b)

Figure 3: The trajectories of tracking errors $e_{1}, e_{2}, e_{3}$ by the proposed method (12) in time period (a) [0,10s] and (b) [5s, $10 \mathrm{~s}$. 


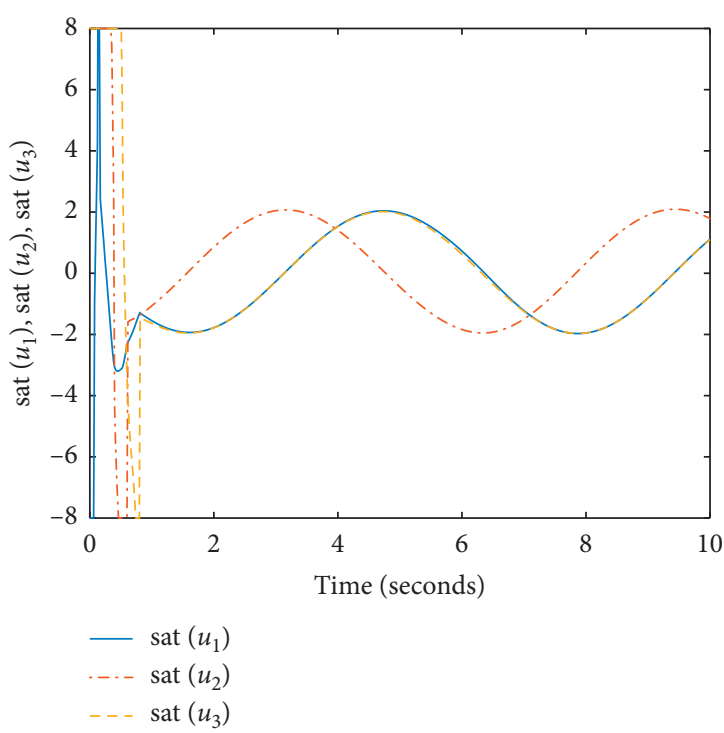

(a)

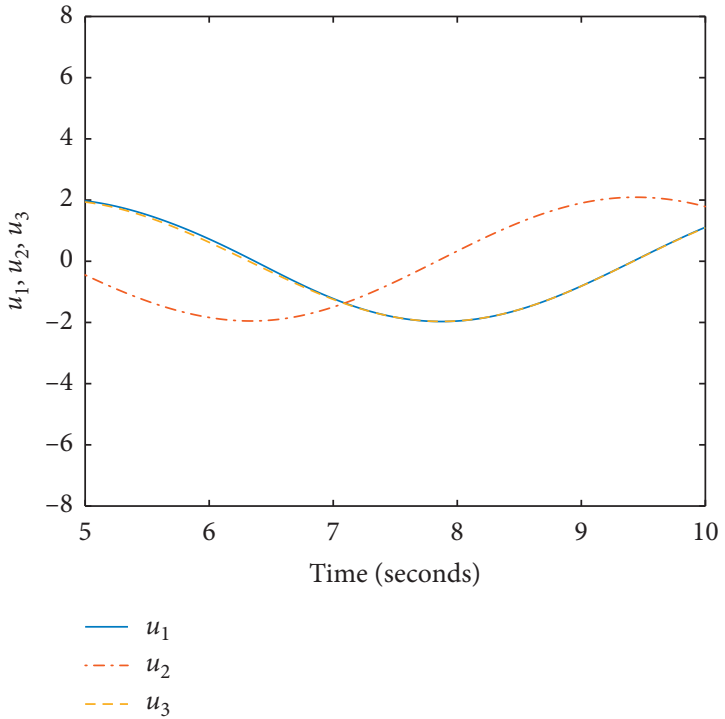

(b)

Figure 4: The trajectories of (a) sat $\left(u_{1}\right)$, sat $\left(u_{2}\right)$, sat $\left(u_{3}\right)$ by method (12) in time period $[0,10 \mathrm{~s}]$ and (b) $u_{1}, u_{2}, u_{3}$ by method (12) in time period $[5 \mathrm{~s}, 10 \mathrm{~s}]$.

where $\zeta=x_{1}, x_{2}, x_{3} ; \xi=y_{1}, y_{2}, y_{3}, k_{1}=1,2,3,4,5 ; k_{2}=$ $1,2,3$.

According to Theorem 2, the ultimate bound of the tracking error $e$ can be estimated in the interval $(-2.4 \times$ $10^{-3}, 2.4 \times 10^{-3}$ ) when $\gamma_{1}=1 / 5$. Now, we give the traditional integral sliding mode control method as follows:

$$
\begin{aligned}
s_{i}= & e_{i}+\int_{0}^{t} \mu_{1} \operatorname{Sign}\left(e_{i}\right) \mathrm{d} \tau, \\
u_{i}= & -\mu_{3} s_{i}-\mu_{4} \operatorname{Sign}^{\gamma_{2}}\left(s_{i}\right)-\widehat{\theta}_{g_{i}}^{T} \varphi_{g_{i}}(y)+\widehat{\theta}_{f_{i}}^{T} \varphi_{f_{i}}(x) \\
& -\mu_{1} \operatorname{Sign}\left(e_{i}\right), \\
\dot{\hat{\theta}}_{g_{i}}= & \eta_{g}\left[s_{i} \varphi_{g_{i}}(y)-\delta_{1} \widehat{\theta}_{g_{i}}\right], \\
\dot{\hat{\theta}}_{f_{i}}= & \eta_{f}\left[-s_{i} \varphi_{f_{i}}(x)-\delta_{2} \widehat{\theta}_{f_{i}}\right],
\end{aligned}
$$

where $\operatorname{Sign}\left(e_{i}\right)=\operatorname{sign}\left(e_{i}\right), \quad \operatorname{Sign}^{\gamma_{2}}(s) i=\left|s_{i}\right|^{\gamma_{2}} \operatorname{sign}\left(s_{i}\right), i=$ $1,2,3$. Obviously, the approximation range of $e$ cannot be obtained directly from (35).

Firstly, by using method (35), the corresponding simulation results are shown in Figures 1 and 2. From Figure 1, it can be found that all three synchronization errors $e_{1}, e_{2}, e_{3}$ can be controlled into an estimated range, however, $\$ e_{-} 1$, e_2\$, and \$e_3\$ appear the chattering phenomenon. Meanwhile, Figure 2 shows that sat $\left(u_{i}\right)$ and $u_{i}$ also appear a large chattering phenomenon, which is mainly caused by the $\operatorname{sign}(\cdot)$ function in method (35). In order to overcome the influence of the sign (.) function, this paper uses the tanh (.) function to design the controller. The simulation results are shown in Figures 3 and 4. From Figure 3, we can find that $e_{1}$, $e_{2}$, and $e_{3}$ are limited within a small range, which is smaller than the estimated range, and there is no chattering phenomenon for $e_{1}, e_{2}$, and $e_{3}$. Finally, comparing Figures 2 and 4 , it can be seen that method (12) is significantly better than method (35) in terms of controller stability. Based on the above discussion, it can be seen that the proposed method (12) in this paper can effectively and quickly synchronize the master system and the slave system, can overcome the chattering phenomenon, and can estimate the predetermined range of synchronization error.

\section{Conclusion}

This paper considered the synchronization problem of a class of finance chaotic systems by using an integrated sliding mode control method. Firstly, the unknown functions $f_{i}(x)$ and $g_{i}(y)$ are estimated by using fuzzy logic systems. Then, a novel integral sliding variable $s_{i}$ is proposed. The proposed control strategy $u_{i}$ and parameter adaptive rules $\widehat{\theta}_{\bar{f}_{i}}$ can ensure that all signals of the closed-loop system are semiglobally uniformly stable, and the ultimate bound of 
the tracking error can be estimated. Comparison results of simulation show that the proposed control method in this paper can ensure that the error fluctuation range is completely within the estimated range and the trajectories of errors and controllers have no chattering phenomenon. Therefore, compared with the traditional integrated sliding mode control method, the proposed control method in this paper has better control performance.

\section{Data Availability}

All datasets generated for this study are included in this paper.

\section{Conflicts of Interest}

The authors declare that there are no conflicts of interest regarding the publication of this paper.

\section{Acknowledgments}

This study was supported by the Key Natural Science Research Projects in Anhui Universities (KJ2019A0695 and KJ2020A0644), the Natural Science Research Projects of Huainan Normal University (2018xj22zd), the Anhui Natural Science Foundation (1608085QA05), and the Major Research Project of Natural Science in Anhui Colleges (KJ2019ZD48).

\section{References}

[1] M. F. Hassan and M. Hammuda, "A new approach for constrained chaos synchronization with application to secure data communication," Journal of the Franklin Institute, vol. 356, no. 12, pp. 6697-6723, 2019.

[2] S. Vaidyanathan and A. T. Azar, "Anti-synchronization of identical chaotic systems using sliding mode control and an application to vaidyanathan-madhavan chaotic systems," in Advances and Applications in Sliding Mode Control Systems, pp. 527-547, Springer, Cham, Switzerland, 2015.

[3] N. Pillai, S. L. Schwartz, T. Ho, A. Dokoumetzidis, R. Bies, and I. Freedman, "Estimating parameters of nonlinear dynamic systems in pharmacology using chaos synchronization and grid search," Journal of Pharmacokinetics and Pharmacodynamics, vol. 46, no. 2, pp. 193-210, 2019.

[4] M. Neubert and M. Fujiwara, Introduction to Chaos: Physics and Mathematics of Chaotic Phenomena, CRC Press, Baco Raton, FL, USA, 2000.

[5] W. Al-Hussaibi, "Effect of filtering on the synchronization and performance of chaos-based secure communication over Rayleigh fading channel," Communications in Nonlinear Science and Numerical Simulation, vol. 26, no. 1-3, pp. 87-97, 2015.

[6] M. Chao and W. Xingyuan, "Hopf bifurcation and topological horseshoe of a novel finance chaotic system," Communications in Nonlinear Science and Numerical Simulation, vol. 17, no. 2, pp. 721-730, 2012.

[7] H. Yu, G. Cai, and Y. Li, "Dynamic analysis and control of a new hyperchaotic finance system," Nonlinear Dynamics, vol. 67 , no. 3, pp. 2171-2182, 2012.

[8] C. Huang and J. Cao, "Active control strategy for synchronization and anti-synchronization of a fractional chaotic financial system," Physica A: Statistical Mechanics and Its Applications, vol. 473, pp. 262-275, 2017.

[9] S. Harshavarthini, R. Sakthivel, Y.-K. Ma, and M. Muslim, "Finite-time resilient fault-tolerant investment policy scheme for chaotic nonlinear finance system," Chaos, Solitons \& Fractals, vol. 132, p. 109567, 2020.

[10] C. Wen and J. Yang, "Complexity evolution of chaotic financial systems based on fractional calculus," Chaos, Solitons \& Fractals, vol. 128, pp. 242-251, 2019.

[11] H. Tirandaz, S. S. Aminabadi, and H. Tavakoli, "Chaos synchronization and parameter identification of a finance chaotic system with unknown parameters, a linear feedback controller," Alexandria Engineering Journal, vol. 57, no. 3, pp. 1519-1524, 2018.

[12] J. A. R. Vargas, E. Grzeidak, and E. M. Hemerly, "Robust adaptive synchronization of a hyperchaotic finance system," Nonlinear Dynamics, vol. 80, no. 1-2, pp. 239-248, 2015.

[13] C. Wang, H. Zhang, W. Fan, and P. Ma, "Adaptive control method for chaotic power systems based on finite-time stability theory and passivity-based control approach," Chaos, Solitons \& Fractals, vol. 112, pp. 159-167, 2018.

[14] J. Keighobadi, M. Hosseini-Pishrobat, and J. Faraji, "Adaptive neural dynamic surface control of mechanical systems using integral terminal sliding mode," Neurocomputing, vol. 379, pp. 141-151, 2020.

[15] X. Chen, J. H. Park, J. Cao, and J. Qiu, “Adaptive synchronization of multiple uncertain coupled chaotic systems via sliding mode control," Neurocomputing, vol. 273, pp. 9-21, 2018.

[16] X.-F. Li, Y.-D. Chu, A. Y. T. Leung, and H. Zhang, "Synchronization of uncertain chaotic systems via completeadaptive-impulsive controls," Chaos, Solitons \& Fractals, vol. 100, pp. 24-30, 2017.

[17] C. Zhou, C. Yang, D. Xu, and C. Chen, "Coexisting attractors, circuit realization and impulsive synchronization of a new four-dimensional chaotic system," Modern Physics Letters B, vol. 33, no. 3, p. 1950026, 2019.

[18] C.-C. Yang and C.-J. Ou, "Adaptive terminal sliding mode control subject to input nonlinearity for synchronization of chaotic gyros," Communications in Nonlinear Science and Numerical Simulation, vol. 18, no. 3, pp. 682-691, 2013.

[19] L. Yang and J. Yang, "Nonsingular fast terminal sliding-mode control for nonlinear dynamical systems," International Journal of Robust and Nonlinear Control, vol. 21, no. 16, pp. 1865-1879, 2011.

[20] R. M. Wang, Y. N. Zhang, Y. Q. Chen, X. Chen, and L. Xi, "Fuzzy neural network-based chaos synchronization for a class of fractional-order chaotic systems: an adaptive sliding mode control approach," Nonlinear Dynamics, vol. 100, pp. 1275-1287, 2020.

[21] C.-S. Chiu, "Derivative and integral terminal sliding mode control for a class of mimo nonlinear systems," Automatica, vol. 48, no. 2, pp. 316-326, 2012.

[22] H. Armghan, M. Yang, A. Armghan, N. Ali, M. Q. Wang, and I. Ahmad, "Design of integral terminal sliding mode controller for the hybrid AC/DC microgrids involving renewables and energy storage systems," International Journal of Electrical Power \& Energy Systems, vol. 119, p. 105857, 2020.

[23] D. Deepika, S. Kaur, and S. Narayan, "Integral terminal sliding mode control unified with UDE for output constrained tracking of mismatched uncertain non-linear systems," ISA Transactions, vol. 101, pp. 1-9, 2020. 
[24] D. Zhao, Q. Zhu, and J. Dubbeldam, "Terminal sliding mode control for continuous stirred tank reactor," Chemical Engineering Research and Design, vol. 94, pp. 266-274, 2015.

[25] H. Liu, Y. Pan, S. Li, and Y. Chen, "Adaptive fuzzy backstepping control of fractional-order nonlinear systems," IEEE Transactions on Systems, Man, and Cybernetics: Systems, vol. 47, no. 8, pp. 2209-2217, 2017. 